\title{
Contemporary Nutrition Interventions to Optimize Performance in Middle-Distance Runners
}

\author{
Trent Stellingwerff \\ Canadian Sport Institute Pacific, Athletics Canada, and University of Victoria \\ Ingvill Måkestad Bovim \\ Norwegian Sports Medicine Centre \\ Jamie Whitfield \\ Australian Catholic University
}

\begin{abstract}
Middle-distance runners utilize the full continuum of energy systems throughout training, and given the infinite competition tactical scenarios, this event group is highly complex from a performance intervention point of view. However, this complexity results in numerous potential periodized nutrition interventions to optimize middle-distance training adaptation and competition performance. Middle-distance race intensity is extreme, with 800 - to $5,000-\mathrm{m}$ races being at $\sim 95 \%$ to $130 \%$ of $\mathrm{VO}_{2} \mathrm{max}_{\text {. }}$ Accordingly, elite middle-distance runners have primarily Type IIa/IIx fiber morphology and rely almost exclusively on carbohydrate (primarily muscle glycogen) metabolic pathways for producing adenosine triphosphate. Consequently, the principle nutritional interventions that should be emphasized are those that optimize muscle glycogen contents to support high glycolytic flux (resulting in very high lactate values, of $>20 \mathrm{mmol} / \mathrm{L}$ in some athletes) with appropriate buffering capabilities, while optimizing power to weight ratios, all in a macro- and microperiodized manner. From youth to elite level, middle-distance athletes have arduous racing schedules (10-25 races/year), coupled with excessive global travel, which can take a physical and emotional toll. Accordingly, proactive and integrated nutrition planning can have a profound recovery effect over a long race season, as well as optimizing recovery during rounds of championship racing. Finally, with evidence-based implementation and an appropriate risk/reward assessment, several ergogenic aids may have an adaptive and/or performanceenhancing effect in the middle-distance athlete. Given that elite middle-distance athletes undertake $\sim 400$ to 800 training sessions with 10-25 races/year, there are countless opportunities to implement various periodized acute and chronic nutrition-based interventions to optimize performance.
\end{abstract}

Keywords: elite, ergogenic aids, nutrition periodization

Middle-distance running events are highly complex from a performance optimization point of view. For example, elite middledistance specialists need to have the aerobic system development approaching marathoners, coupled with some of the mechanical properties of elite sprinters, while concurrently having worldclass anaerobic capacities with polished tactical race instincts (Hanley \& Hettinga, 2018; Sandford et al., 2018). These performance requirements result in highly complex training approaches, with significant differences within and between athletes throughout macro- (yearly/monthly) and micro- (weekly/within-day) cycle periods (Martin \& Coe, 1991). Accordingly, given that elite middle-distance athletes undertake $\sim 400$ to 800 unique yearly training sessions, and race 10-20 times per year (personal observations),

Stellingwerff is with the Canadian Sport Institute Pacific, Victoria, British Columbia, Canada; Athletics Canada, Ottawa, Ontario, Canada; and the Dept. of Exercise Science, Physical and Health Education, University of Victoria, Victoria, British Columbia, Canada. Bovim is with Norwegian Sports Medicine Centre, Oslo, Norway. Whitfield is with Exercise and Nutrition Research Program, Mary MacKillop Institute for Health Research, Australian Catholic University, Melbourne, Victoria, Australia. Address author correspondence to Trent Stellingwerff at tstellingwerff@csipacific.ca. there are countless opportunities to implement various periodized acute and chronic nutrition-based interventions. Therefore, the purpose of this review is to provide an evidence-based update since the last International Association of Athletics Federations (IAAF) consensus meeting (Stellingwerff et al., 2007) on contemporary nutrition recommendations to optimize adaptation to training and enhance competition performance in elite middle-distance athletes. The focus of this review will be on IAAF events ranging from 800 to $5,000 \mathrm{~m}$, termed middle-distance events hereafter. Where possible we will integrate practical recommendations coupled with peer-reviewed data to support our nutritional recommendations. However, given the event group complexity and the numerous potential interventions, many of the other IAAF papers will be referenced, and this review will focus exclusively on key novel interventions for middle-distance athletes.

\section{Physiological and Bioenergetic Determinants of Middle-Distance Success}

The complexity of middle-distance running performance determinants includes physiological aspects such as bioenergetics/energy 
systems (Duffield et al., 2005; Spencer \& Gastin, 2001), but most certainly also includes elements such as biomechanics/structural (force, stride frequency, body mass [BM]) aspects (Weyand \& Davis, 2005) as well as numerous sociological and psychological constructs. However, the majority of nutritional-based interventions that impact middle-distance performances are bioenergetically based and, thus, will be the determinants of performance focus in this article (Figures 1 and 2). For individuals interested in nutrition interventions in events of a biomechanical and structural performance origin, please see Sygo et al. (2018). In order to understand how these interventions may relate to the energetic demands of a given exercise bout, it is imperative to understand how the various metabolic pathways interact in order to produce the required amounts of adenosine triphosphate (ATP). This understanding best informs potential nutritional interventions that may impact training adaptation and/or performance in a periodized manner. This is especially important in middle-distance events since they rely heavily on both substrate-level phosphorylation (anaerobic) and oxidative phosphorylation (aerobic) ATP production. The energy system aspects of middle-distance running has been well described previously (Duffield et al., 2005; Spencer \&
Gastin, 2001), and only key elements will be described here (Figure 1).

Middle-distance race intensity is extreme, with 800- to 5,000-m races being at $\sim 95 \%$ to $130 \%$ of $\mathrm{VO}_{2} \max$ (Duffield et al., 2005), or $75-85 \%$ of maximum sprint speed (Figure 1). Given these race intensities and required training intensities, middle-distance athletes have a high number and highly developed Type IIa/IIx (intermediate) fiber morphology (Costill et al., 1976) and rely almost exclusively on carbohydrate $(\mathrm{CHO}$; primarily muscle glycogen) and phosphocreatine metabolic pathways for producing ATP. Accordingly, most middle-distance athletes can generate peak lactate values over $20 \mathrm{mmol} / \mathrm{L}$ resulting in muscle $\mathrm{pH}$ values as low as 6.6 (Hermansen \& Osnes, 1972). Therefore, middledistance athletes have highly refined anaerobic capacities, or tolerance, which is certainly a product of highly developed intermediate Type IIa (fast oxidative) fiber types, which are especially high in muscle carnosine concentrations. Carnosine is an undisputed $\mathrm{pH}$ buffer contributing as much as $15 \%$ to total muscle buffering capacity, as it has long been known that sprinters and rowers have nearly double the amount of muscle carnosine than marathon runners, strongly correlating to their Type II muscle fiber

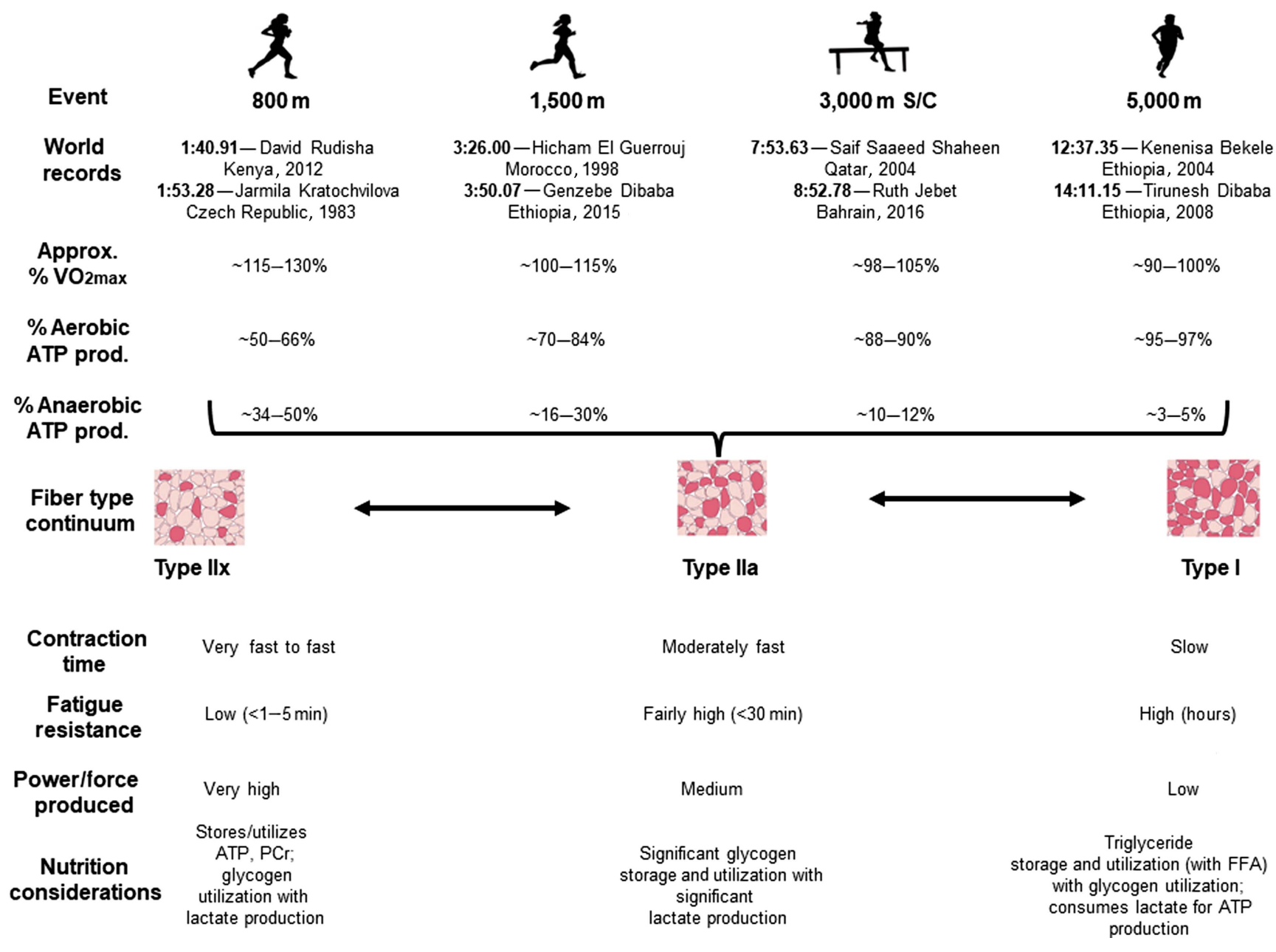

Figure 1 - Differences in ATP energy source provision in middle-distance events within the context of fiber types and endogenous nutrition considerations. It should be noted that fiber typing is a continuum, and only 100- to 400-m athletes have >60\% Type IIx fiber types, while most middledistance athletes Type I fibers range from $40 \%$ to $70 \%$ (Costill et al., 1976). ATP = adenosine triphosphate; FFA = free fatty acids; $\mathrm{PCr}=$ phosphocreatine; prod. = production. Data adapted from Astrand et al. (1986), Costill et al. (1976), Gaston (1998), and Spencer and Gastin (2001). 


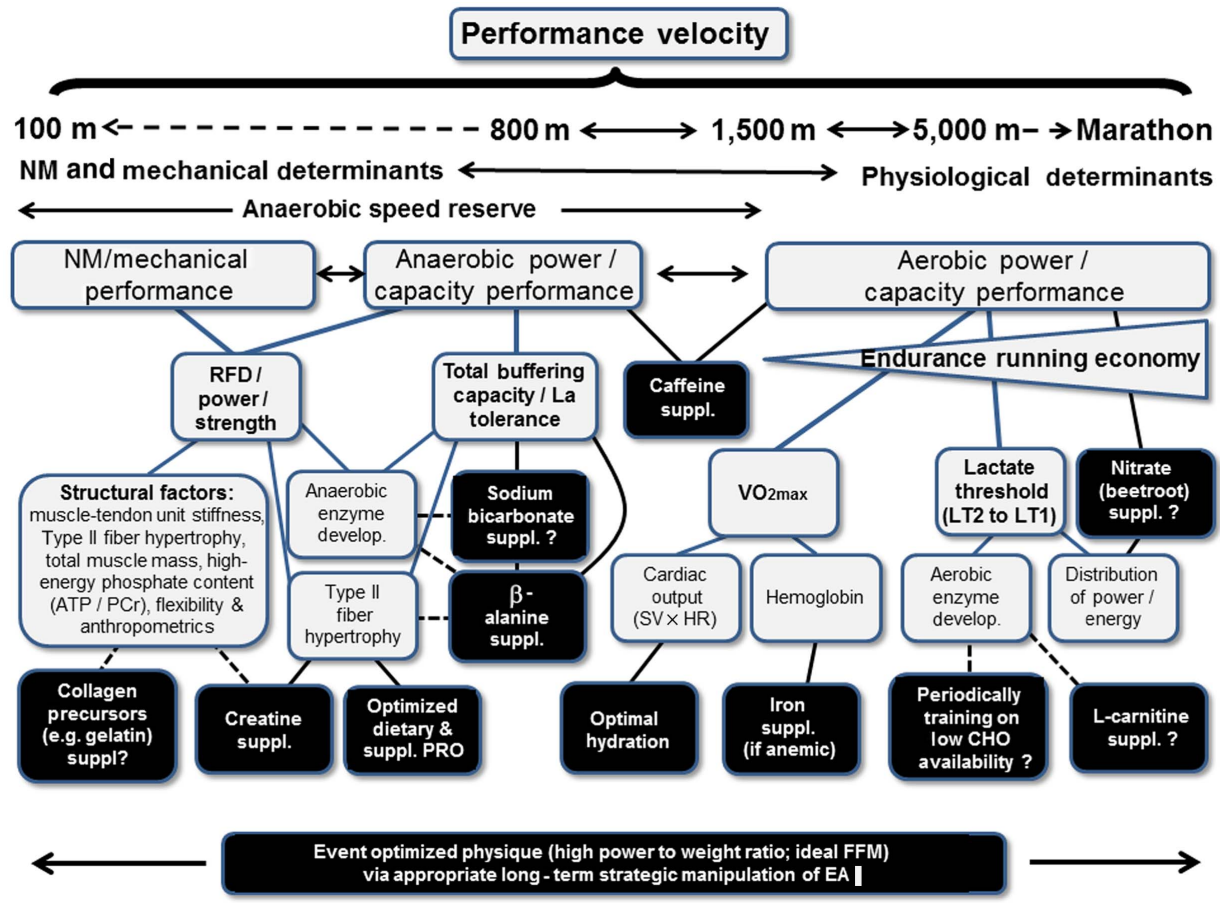

Figure 2 - Framework of the interactions between the structural and physiological determinants of performance (in white boxes) and potential nutritional interventions (black boxes) in middle-distance athletes. It should be highlighted that performance determinates are a continuum, with middledistance athletes featuring both structural and physiological elements. Some nutrition interventions are acute (e.g., caffeine), while others are chronic in combination with training (e.g., creatine supplementation). A dotted line indicates a potential training-induced nutritional adaptation. A question mark (?) highlights the requirement for more scientific validation. $\mathrm{ATP}=$ adenosine triphosphate $\mathrm{CHO}=$ carbohydrate; $\mathrm{EA}=$ energy availability; FFM $=$ fat-free mass; $\mathrm{HR}=$ heart rate; $\mathrm{La}=$ lactate; $\mathrm{LT}=$ lactate threshold; $\mathrm{NM}=$ neuromuscular; $\mathrm{PCr}=$ phosphocreatine; $\mathrm{PRO}=$ protein; $\mathrm{RFD}=$ rate of force development; suppl. = supplementation; $\mathrm{SV}=$ stroke volume; $\mathrm{VO}_{2} \mathrm{max}=$ maximal oxygen consumption .

morphology (Parkhouse et al., 1985). However, when considering the entire spectrum of middle-distance race durations $(\sim 1.5$ to $15 \mathrm{~min})$, the majority of middle-distance events are highly aerobic and require significant aerobic capacity development, as the measured aerobic contributions from 800 to $5,000 \mathrm{~m}$ range from $\sim 50 \%$ to $95 \%$ (Figure 1). Indeed, the majority of aerobic ATP production during intense exercise comes from glycolysis (primarily glycogen breakdown) and the aerobic disposal of pyruvate via the enzyme pyruvate dehydrogenase into the mitochondria (Parolin et al., 1999). There is also very large variability between athletes in aerobic versus anaerobic ATP contribution, especially over the 800 -m event (1.5-2 min), with anaerobic contributions to the 800$\mathrm{m}$ event ranging from $19 \%$ to $48 \%$, depending on the fitness of the subject and methods used (Duffield et al., 2005; Spencer \& Gastin, 2001). A lot of this variability is probably due to the fact that middle-distance racing also involves nearly peak speed running (Sandford et al., 2018), which is more impacted by biomechanics and structural constraints (Weyand et al., 2005) outlined in Figure 2, including the key emerging concept of anaerobic speed reserve (Buchheit \& Laursen, 2013). Anaerobic speed reserve is the difference between an athlete's maximal sprint speed and velocity at $\mathrm{VO}_{2}$ max, featuring structural/biomechanical considerations for maximal sprint speed as well as energy system interventions for velocity at $\mathrm{VO}_{2} \max$ (Figure 2).

Bioenergetics during racing is exponentially more complicated when considering the infinite tactical situations. For example, middle-distance events are not run in lanes and feature drafting (drafting in still wind at middle-distance speeds has been shown to reduce $\mathrm{VO}_{2}$ cost by $\sim 2 \%$ to $4 \%(\sim 0.3$ to $1.0 \mathrm{~s} / \mathrm{lap}$; Pugh, 1971$)$ and constant tactical decision making, which affect aerobic versus anaerobic components and the anaerobic speed reserve continuum. Furthermore, since middle-distance events are run at such high intensities, there is very little room for tactical errors because they come at such a high metabolic cost. Therefore, aerobic and anaerobic pathways need to produce remarkable rates of ATP for middle-distance success. Accordingly, from a bioenergetic perspective, optimizing muscle glycogen contents to support high glycolytic flux (resulting in very high lactate values) with appropriate buffering capabilities, while optimizing power to weight ratios, are the principle nutritional interventions to emphasize in middle-distance runners (Figure 2).

\section{Periodized Nutritional Strategies to Support Periodized Training}

The concept of nutrition periodization has been emerging as a key construct to optimize sports-specific nutrition recommendations (Jeukendrup, 2017; Stellingwerff et al., 2007, 2011), with most of these papers focusing on macronutrition periodization. More recently the concept of dietary microperiodization (weeks to within-day) has been termed, which examines the temporal associations between specific training stimuli, daily life demands and associated nutrition choices (Heikura et al., 2017a). This section will focus on novel macro- to micronutrition periodization interventions in middle-distance athletes, with further nutrition 
periodization recommendations made by Morton et al. (Stellingwerff et al., 2018).

\section{Macroperiodization (Months to Weeks) Nutrition Recommendations}

Theoretical guidelines for seasonal macroperiodization of nutrition for middle-distance runners across their periodized plans have previously been presented (Stellingwerff et al., 2007, 2011), and data on typical dietary intakes of middle-distance athletes are featured here (Heikura et al., 2017a, 2017b; 2018). Most elite middle-distance runners train like marathoners during the general preparation phase, with high, but highly variable, volumes during the fall/winter ( 40 to $180 \mathrm{~km} /$ week). Subsequently, training gradually shifts throughout the season to higher intensities/lower volumes and major anaerobic-based sessions toward the peak season. Therefore, the optimal nutrition for an athlete will vary considerably in amount (calories) and type (macronutrient profile) in conjunction with the phase-specific training demands. Since the majority of middle-distance training is performed at or above $75 \% \mathrm{VO}_{2} \mathrm{max}$, and this dependency on CHO-based ATP provision increases throughout the training year toward a championship peak, $\mathrm{CHO}$-rich foods must provide the majority of the energy provision. Accordingly, a habitually high $\mathrm{CHO}$ diet with 7-10 g $\mathrm{CHO} \cdot \mathrm{kg} \mathrm{BM}^{-1} \cdot \mathrm{day}^{-1}$ is recommended to restore glycogen stores, with protein $(\mathrm{PRO})$ intake during hard training phases at $\sim 1.5$ to $1.7 \mathrm{~g} \mathrm{PRO}^{-1} \cdot \mathrm{kg} \mathrm{BM}^{-1} \cdot \mathrm{day}^{-1}$, but substantially less $\mathrm{CHO}$ is required on easy days, and during rest and recovery phases. There have been no major alternations to these original recommendations (Stellingwerff et al., 2007, 2011); instead, this review will focus on novel approaches.

Regarding the appropriate macroperiodization of calories, a research field that has received much recent attention has been the relative energy deficiency in sport (RED-S) and the impact that chronic energy availability (EA) can have on health, body composition, and performance (reviewed here: Melin et al., 2018). Macroperiodization of EA (defined as energy intake minus exercise energy expenditure) underpins the optimization of body composition periodization. In terms of middle-distance athlete health, a very recent multicenter/multicountry paper ( $n=59$ athletes) examined relative energy deficiency in sport indicators, prevalence, and symptoms. This paper demonstrated that amenorrheic females (37\% of females) and males in the lowest quartile of testosterone $(15.1 \pm 3.0 \mathrm{nmol} / \mathrm{L} ; 42 \%$ of males) had 10-fold higher missed training days and 4.5 times the number of bone injuries than the rest of the athletes (Heikura et al., 2018). Given that the likelihood of achieving a performance goal decreases sevenfold in track and field athletes over a 5-year period when completing less than $80 \%$ of planned training weeks (Raysmith \& Drew, 2016), the impact that optimal dietary EA can have on health and performance of middle-distance runners is profound.

However, a key performance indicator for elite middledistance runners is having a very high power to weight ratio, which features elite athletes who have very low levels of body fat during peak championship season, which may result in undesirable relative energy deficiency in sport outcomes. Therefore, it is not sustainable from a health perspective to be at peak body composition year-round, so body composition needs to be strategically periodized. The limited published body composition ranges suggest elite female middle-distance runners are $\sim 8 \%$ to $12 \%$ body fat ( $\sim 0$ to $60 \mathrm{~mm}$ for International Society for the Advancement of Kinanthropometry sum of 8 ) and males are $4 \%$ to $6 \%$ body fat
( $\sim 30$ to $40 \mathrm{~mm}$ International Society for the Advancement of Kinanthropometry sum of 8 ) in peak competition season (Fleck, 1983). However, very little scientific information exists on how to optimally implement interventions around body composition periodization throughout a given year or over a career. A recent case study featured the body composition of an Olympiclevel female middle-distance runner throughout a 9-year international career (Stellingwerff, 2018). During the general preparation phase, the athlete was at $\sim 2 \%$ to $4 \%$ over ideal competition phase BM with optimal EA being prioritized. In the lead-up to the competition phase, body composition was optimized by creating an individualized time frame and mild caloric deficit ( $-300 \mathrm{kcal})$. As a result, significant and purposeful seasonal fluctuations were observed in anthropometric outcomes between training phases. Importantly, the athlete only suffered two injuries over the 9-year follow-up. Despite a strong conceptual underpinning, more research is needed on the optimal implementation of periodized body composition strategies in short- and long-term planning.

\section{Microperiodization (Week to Within-Day) Nutrition Recommendations}

Many elite middle-distance athletes can undertake two to three training sessions per day (e.g., track-specific interval session, easy off track run, and weights), all of which are higher intensity; thus, optimizing recovery (muscle glycogen and PRO resynthesis) between sessions is a primary objective if training quality is to be maintained. Furthermore, high-intensity training can result in appetite suppression (Hazell et al., 2016), which might impact ad libitum caloric intake. To optimize nutrition around training and competition, athletes need to plan their days well, as some athletes will be away from home the entire day due to school and/or work. Thus, having portable and quickly accessible high-quality nutrition and hydration is sometimes the largest challenge facing athletes. From a practical perspective, it is important for nutrition practitioners to not just give nutritional education/advice but also actively assess if the athlete is able to actually implement the recommendations into their daily routines, as there can be a mismatch between nutrition advice and athlete practice. In support of this, several recent studies conducted primarily in a large cohort of elite middle-distance athletes $(n=38)$ were surveyed on their nutritional knowledge. Interestingly, most athletes reported to have sound recovery nutrition knowledge, as they said they focused on adequate fueling $(96 \%)$ and $\mathrm{CHO}$ and PRO recovery (87\%) around key training sessions (Heikura et al., 2017b). However, when this same cohort of athletes undertook 7 days of dietary recording and assessment, on average, no males or females actually reached the recommended postworkout PRO intake levels, and only males on hard training days reached the CHO recovery recommendations (Heikura et al., 2017a). Therefore, despite these athletes having decent nutrition recovery knowledge, most athletes did not systematically follow the most recent sports nutrition guidelines to optimize recovery.

It is beyond the scope of this review to cover the daily $\mathrm{CHO}$ and PRO recommendations in depth. In terms of within-day macronutrient microperiodization, PRO is especially important to not only optimize acute recovery, but daily overall muscle/ body PRO synthesis and adaptation (for review see: Phillips et al., 2018). Furthermore, there is growing evidence that strategically manipulating acute within-day $\mathrm{CHO}$ availability can serve as a potent mediator in the adaptive response to endurance training, of which the interested reader should be referred (Impey et al., 2018; Stellingwerff et al., 2018). 


\section{Optimizing Competition Nutrition}

Most elite middle-distance athletes will race between 10 and 25 times per year with substantial traveling to meets throughout the global IAAF circuit. Most of these meets are "one-off" races, with athletes flying the day or two before the meet and leaving the day after competition. Considerations around travel fatigue, jet lag, and racing fatigue (emotional/physical) must all be considered (Table 1).

In the weeks prior to major championships, athletes start tapering, resulting in significantly reduced training $(\sim 30 \%$ to $60 \%$ ) and exercise energy expenditure, coupled with staying in prechampionship training camps or in an athlete's village. All of this requires significant travel, which brings a whole host of associated nutritional challenges beyond the scope of this review (see Halson et al., 2018). Conversely, for some athletes entering competition phase, race anxiety may affect their appetite cues and result in significantly reduced energy intake. It should be noted that our scientific understanding of the impact of changes in acute exercise energy expenditure (e.g., tapering) and competition anxiety on energy intake and appetite cues in athletes is poorly understood and rife for further scientific exploration.

To optimize either race day nutrition or major championship nutrition choices, the athlete needs to be motivated to make an individualized plan, of which all factors for consideration are highlighted in Table 1. Although a single race is unlikely to exhaust fuel stores for middle-distance runners, athletes competing many times over a championship or over the season, coupled with excessive travel fatigue, can take a physical and emotional toll, of which proactive and integrated nutrition planning can have a profound recovery effect over a long race season.

\section{Ergogenic Aids for Middle-Distance Runners}

The physiological and bioenergetic determinants of performance for middle-distance running tend to be the focus of most training plans as well as targets for nutritional interventions and dietary ergogenic aid/supplementation (Figure 2, black intervention boxes). Unfortunately, while there are countless commercially available supplements that promise to improve performance, very few have been validated by scientific studies (Peeling et al., 2018). The focus of this section will therefore be on those supplements that both have an evidence-based support and second, ones that are applicable to middle-distance runners and published since the last consensus (Table 2).

\section{Caffeine}

Caffeine is a natural central nervous system stimulant that has many proposed effects relevant for performance, including improved neuromuscular function, increased alertness, and reduced fatigue and ratings of perceived exertion (RPE) during exercise (Burke, 2008). The summary of evidence suggests that best practice is supplementation with $3-6 \mathrm{mg} / \mathrm{kg} \cdot \mathrm{BM}$ consumed $\sim 60 \mathrm{~min}$ prior to exercise. However, there is also growing interest in the use of low $(\leq 3 \mathrm{mg} / \mathrm{kg} \mathrm{BM})$ doses of caffeine as this may maximize any performance-enhancing effects while simultaneously minimizing the risk of negative side effects including anxiety, insomnia, and restlessness associated with larger doses (Spriet, 2014). In addition to traditional methods of delivery such as coffee and caffeine capsules/tablets, there is an increasing amount of research focused on alternative delivery methods such as caffeinated gum (Wickham \& Spriet, 2018), which may also be relevant for in-competition use. A recent systematic review (Ganio et al., 2009) addressed the effect of caffeine on endurance performance, including 33 sport-specific trials of varying modalities and duration. In the context of middle-distance running, four of these studies were of a relevant duration ( 2 to $15 \mathrm{~min})$ and displayed a $\sim 1.1 \pm 0.3 \%$ improvement over placebo. This is consistent with findings in well-trained club-level runners (Table 2), as studies have shown a 1-2\% improvement in time-trial (TT) performance over both 1 mile (Clarke et al., 2018) and 5,000 m (O'Rourke et al., 2008). A recent meta-analysis (Christensen et al., 2017) also found a small but meaningful ( $\mathrm{ES}=0.41, P=.002)$ effect of caffeine supplementation on exercise speed in performance tests lasting 0.75 to $\sim 8 \mathrm{~min}$. Taken altogether, the evidence suggests that caffeine may be useful for improving performance across the entire spectrum of middle-distance events.

\section{Inorganic Nitrate}

Nitric oxide (NO) is a potent signaling molecule that targets and affects multiple tissues, including skeletal muscle. While NO can be produced endogenously via NO synthases, supplementation with inorganic nitrate in a variety of forms (the most common of which are nitrate salts, beetroot juice) can also increase whole-body NO bioavailability. In recent years, nitrate supplementation has gained popularity due to early studies demonstrating improvements in exercise efficiency (decreased $\mathrm{O}_{2}$ cost at the same absolute workload) and a reduction the $\mathrm{VO}_{2}$ slow component (which reflects a loss in muscle efficiency during high-intensity exercise), following both acute and chronic supplementation (reviewed in Jones, 2014). Recent work has also demonstrated that nitrate can also improve high-intensity exercise performance, possibly due to enhanced function of Type II muscle fibers (Bailey et al., 2015), and there is a growing field examining the effects of nitrate in unique environmental conditions such as altitude, of which many elite middle-distance athletes implement into their programs (Shannon et al., 2017b). Given its purported exercise-related effects, nitrate presents as an attractive candidate for enhancing performance across all middle-distance events. A recent study examined the effects of both acute and chronic beetroot juice supplementation compared with a nitrate-depleted beetroot juice placebo in elite 1,500-m runners (personal best $3 \mathrm{~min} 56 \mathrm{~s}$; $\mathrm{VO}_{2}$ peak: $80 \pm 5 \mathrm{ml} \cdot \mathrm{min}^{-1} \cdot \mathrm{kg} \mathrm{BM}^{-1}$; Boorsma et al., 2014). Despite large increases in plasma nitrate in both the acute and chronic trials compared with the placebo condition, submaximal running $\mathrm{VO}_{2}$ and subsequent $1,500-\mathrm{m}$ TT performance was unaffected (Table 2). In contrast, Shannon et al. (2017a) demonstrated a $1.9 \%$ improvement in 1,500-m treadmill TT performance, albeit in a less trained population $\left(\mathrm{VO}_{2}: 62 \pm 8 \mathrm{ml} \cdot \mathrm{min}^{-1} \cdot \mathrm{kg} \mathrm{BM}^{-1}\right)$. Overall, these findings are consistent with a number of other studies that have failed to demonstrate an improvement in exercise economy in elite and/or well-trained athletes (Jones, 2014). Indeed, a recent meta-analysis that included a subgroup analysis of well-trained/ elite athletes showed no significant effect $(\mathrm{ES}=-0.04, P=.8$; Van De Walle \& Vukovich, 2018), while a meta-analysis focusing on TTs found a slight, but trivial difference $(\mathrm{ES}=0.19, P=.09)$ following nitrate supplementation compared with placebo (Christensen et al., 2017). At present, it is unclear why elite athletes respond differently to supplementation; however, it is possible that a higher basal concentration of nitrate and nitrite and/or adaptation 


\section{Table 1 Nutritional Challenges and Solutions Facing Elite Middle-Distance Runners Travelling and at Major Competitions}

\section{Nutrition challenges \\ (from least to most important)}

Travel

Food choices at event/ championship

Recovery during multiple races/rounds

Race-day nutrition

\section{Challenges}

The time for ground transportation and long-haul flights can sometimes mean $24+\mathrm{hr}$ of travel.

The timing of meals is an important consideration for travel fatigue and jet-lag symptoms.

Long-haul flights are very dry and increase the risk for athlete illness.

Athletes do not have direct influence on what food is served at events.

All you can eat buffets (e.g., Olympic Village) are often the norm and boredom and/or stress eating can easily occur.

At rest days between races or at pre-championship camps, the energy output is less than when in normal training.

Consider potential transportation delays back to the hotel/village or an athlete being selected for a doping test at the stadium or prolonged media requests that will interfere with the optimal recovery plan.

Middle-distance runners who initiate racing with low muscle glycogen will not perform optimally (Maughan \& Poole, 1981).

Do not let race stress/anxiety dictate over- or undereating.

Racing late in the evening.

Racing early in the morning.

Athletes can experience stomach issues or diarrhea prior races due to nervousness.

The circuit and championships are located all over the world, with related differences in food options.

Hydration in usual hot climates during competition season.

Timing of prerace snacks and ergogenic aids are important.

Race tactics is crucial for optimal performance.

Middle-distance athletes start their warm-up more than $60 \mathrm{~min}$ before the start of the race and need to stay hydrated and fueled before they get to the start line.

\section{Nutrition solutions/considerations}

Individualized and optimal nutrition/hydration availability is key. Understand the travel logistics and food options available while traveling and plan ahead to bring any food/fluids the athlete may need.

An athlete's eating and drinking pattern is also a zeitgeber for circadian readjustment; so try to eat and drink to the new time zones meal pattern as soon as possible upon arrival (Reilly et al., 2007).

Remember to drink enough fluids on longer flights, both to stay hydrated and to keep mucous moist for optimal function and avoidance of air-borne viruses (Halson et al., 2018).

Plan ahead by knowing what will be served and augmenting choices with one's own food.

Having an individualized nutrition plan to help circumvent this.

It has been shown that ad libitum energy intake is not immediately matched by reduced energy expenditure (Stubbs et al., 2004). Therefore, athletes should microperiodize with less energy intake when not training hard or during the taper to maintain an ideal peak body composition.

Bring recovery products to the stadium to allow for optimal recovery timing. Have a clear plan for media requests and timing during the rounds of a championship.

Optimization of recovery, and specifically $\mathrm{CHO}$, is fundamental to race performance in later rounds of a championship. Athletes should aim for large amounts of exogenous carbohydrate (1-1.5 $\mathrm{g} \cdot \mathrm{kg}^{-1} \cdot \mathrm{hr}^{-1}$; Jentjens \& Jeukendrup, 2003) and $0.3 \mathrm{~g} / \mathrm{kg}$ of intact protein in the hours after the race (Moore et al., 2009).

Athletes should make a plan for their entire race day, that includes a meal plan.

Make a plan to implement meal timing for later evening races; ideally practice it during training or smaller races. Hydrate appropriate to the weather.

Get up $\sim 3$ to $4 \mathrm{hr}$ prior to race to allow the body to wake up and get a carbohydrate-rich breakfast to fill up the liverglycogen stores. Hydrate appropriate to the weather.

Eat well-tolerated foods, and ones the athlete prefers. Stick to easily digested carbohydrate and some protein-rich food.

Consider food options that are easily available worldwide. The prerace meal should be high in carbohydrates and consumed 1-6 hr before competition.

Drink enough during the day. Last $60-120$ min before the warm-up athletes should aim to drink $400-600 \mathrm{ml}$ water or sports drink.

Eat last meal 1-4 hr prior the warm-up. And follow the guidelines for caffeine, bicarbonate, or nitrate as discussed in this paper.

Carbohydrate intake during warm-up can give the runners neuromuscular support via the attenuation of cognitive fatigue that can reduce technical and tactical errors as shown in soccer players (Currell et al., 2009).

Bring sports drink for the warm-up, as even carbohydrate mouth rinsing has been shown to have performanceenhancing effect (Stellingwerff \& Cox, 2014). 


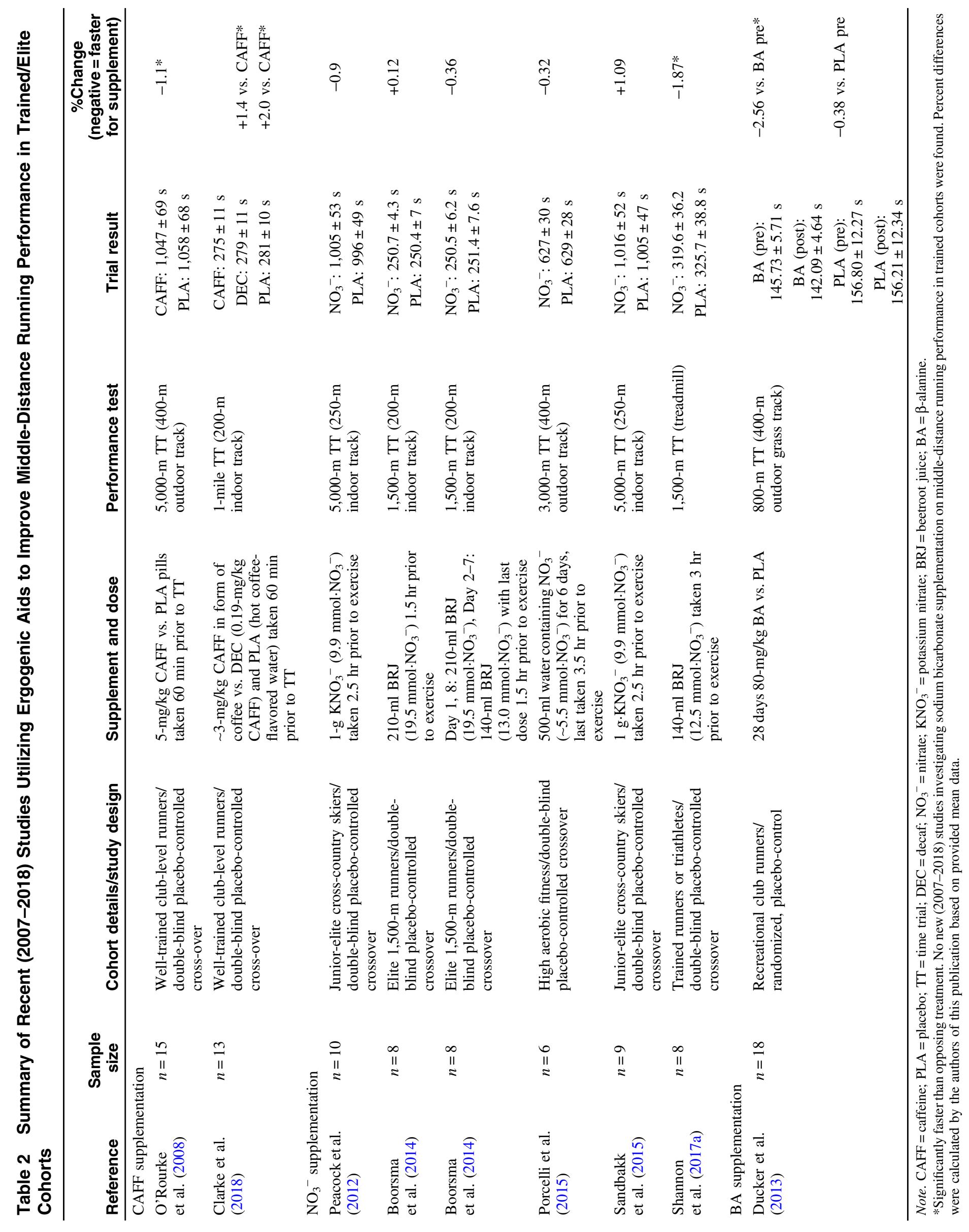


to several years/decades of training negates any further effect given via nitrate supplementation.

\section{$\beta$-Alanine-Intracellular Buffering}

Carnosine is an intracellular dipeptide found in a variety of tissues, including skeletal muscle, and has a variety of proposed physiological roles including acting as an intracellular buffer (Harris \& Stellingwerff, 2013) and, more recently, as an intracellular shuttle of hydrogen cation $\left(\mathrm{H}^{+}\right)$and calcium $\left(\mathrm{Ca}^{2+}\right)$ from the sarcoplasmic reticulum to the actin-myosin cross-bridge locations within the muscle (Blancquaert et al., 2015; Swietach et al., 2014). Carnosine is produced in muscle via the combination of the amino acids Lhistidine and $\beta$-alanine (BA), with dietary intake of the latter amino acid limiting synthesis (Harris et al., 2006). High-intensity exercise causes an accumulation of $\mathrm{H}^{+}$, which is involved in the drop of intracellular $\mathrm{pH}$ from $\sim 7.0$ to $\sim 6.6$ (Hermansen \& Osnes, 1972) which could potentially be one of the contributing factors to fatigue (although $\mathrm{H}^{+}$is certainly not the only reason for changes in muscle $\mathrm{pH}$ and fatigue; Gladden, 2004). As such, supplementation with $\mathrm{BA}$ resulting in increased muscle carnosine presents an opportunity to increase skeletal muscle $\mathrm{pH}$ buffering capacity and/or shuttling of $\mathrm{H}^{+}$and $\mathrm{Ca}^{2+}$, thereby potentially delaying the onset of fatigue and improving performance (Saunders et al., 2017a). Research has demonstrated that supplementation can increase carnosine concentration equally in both Type I and Type II fibers in the range of 40-80\% depending on dose and timing (Stellingwerff et al., 2012). Furthermore, recent data (Saunders et al., 2017b) demonstrate that chronic supplementation ( 24 weeks with $6.4 \mathrm{~g}$ /day BA) can result in further increases beyond those seen with a more typical 2- to 4-week loading period (skeletal muscle carnosine had increased by $-51 \%$ by Week 4 in contrast to the $90-95 \%$ increase seen by Weeks 20-24). Importantly, the additional increase in carnosine content at Week 20 and 24 coincided with an increase in the likelihood of positive improvement in time to exhaustion $(\mathrm{ES}=0.62,1.21$, and 0.83 at Weeks 4, 20, and 24, respectively). Therefore, while current recommendations are daily consumption of $\sim 3$ to $6 \mathrm{~g} \mathrm{BA}$ for a minimum of 2-4 weeks (Saunders et al., 2017a), longer periods may result in additional increases in skeletal muscle carnosine content. In the context of middle-distance running performance, athletes running 800-1,500 $\mathrm{m}$ are the most likely to benefit from BA supplementation, as compared with 5,000-m athletes, as a recent meta-analysis demonstrates that the strongest effect is on exercise lasting 60-240 s (Hobson et al., 2012; Saunders et al., 2017a). This has previously been demonstrated in recreational club runners, as 28 days of supplementation with BA improving 800-m running performance by $2.6 \%$ (Table 2; Ducker et al., 2013). However, despite the well-established evidence demonstrating the ability to increase skeletal muscle carnosine stores through BA supplementation, the results of several meta-analyses suggest that the effects of BA are stronger on exercise capacity tests (e.g., time to exhaustion), as opposed to specific performance tests (Christensen et al., 2017; Hobson et al., 2012; Saunders et al., 2017a). Furthermore, effects appear to be stronger in nontrained recreationally active individuals (Saunders et al., 2017a), with smaller, but potentially meaningful, effect sizes in well-trained and elite athletes (possibly due to the smaller overall number of studies performed in this population). Consequently, it is difficult to definitively say whether BA supplementation will improve performance in elite middle-distance athletes, given the lack of data in this cohort, the prevalence of nonperformance related tests and the smaller effect sizes. However, given the absence of side effects and the potentially meaningful improvements in performance outlined previously, individual athletes and their support teams may consider trialing BA supplementation to determine if it is effective for them.

\section{Sodium Bicarbonate-Extracellular Buffering}

Sodium bicarbonate $\left(\mathrm{NaHCO}_{3}{ }^{-}\right)$is a key extracellular buffer, which can improve performance by increasing extracellular bicarbonate (concentrations and blood $\mathrm{pH}$. In doing so, the efflux of lactate and $\mathrm{H}^{+}$cations out of skeletal muscle is increased thereby minimize intracellular metabolic perturbations linked to fatigue (Jubrias et al., 2003). As with BA, research has typically focused on high-intensity exercise lasting 60-360 s (800-1,500 m) where $\mathrm{H}^{+}$accumulation and decreases in both intra- and extracellular $\mathrm{pH}$ are most likely to occur. While the timing and ingestion patterns vary greatly between studies, it has been suggested that a $5-6 \mathrm{mmol} / \mathrm{L}$ increase in blood bicarbonate concentration is required to improve performance (Carr et al., 2011a). As a result, current guidelines suggest supplementing with 0.2-0.4 g/kg BM 60-150 min prior to exercise (Carr et al., 2011a). A recent meta-analysis by Christensen et al. (2017) found a small but meaningful $(\mathrm{ES}=0.40, P<.001)$ effect of $\mathrm{NaHCO}_{3}{ }^{-}$supplementation on exercise speed in TT-based performance tests indicating it can improve intense endurance performance. These findings are supported by other published work assessing a broader scope of performance measures and protocols demonstrating a moderate effect of $\mathrm{NaHCO}_{3}{ }^{-}$on performance outcomes $(\mathrm{ES}=0.41, P=.007)$, although it is worth noting the effects were greater in untrained versus trained participants (Peart et al., 2012). Classical work also supports use of $\mathrm{NaHCO}_{3}{ }^{-}$for improving running performance, as both 800- (Wilkes et al., 1983) and 1,500-m (Bird et al., 1995) performance was improved in trained runners compared with placebo and control. It is however important to note that some individuals suffer from gastrointestinal (GI) upset following supplementation with $\mathrm{NaHCO}_{3}{ }^{-}$, particularly when consuming doses greater than $0.3 \mathrm{~g} / \mathrm{kg} \mathrm{BM}$ (Carr et al., 2011b) and that individuals experiencing GI upset do not improve performance postsupplementation (Price \& Simons, 2010; Saunders et al., 2014). Runners may be particularly sensitive to these issues given the nature of the sport (upright posture and prone to jostling of fluids in the stomach), and thus, strategies such as consuming the supplement with food (Carr et al., 2011b) may prove beneficial to minimize adverse effects. An additional concern that is of particular relevance for weight-dependent runners is the potential for increased fluid retention, and therefore an increase in BM, as a result of the increased sodium intake (Sims et al., 2007a, 2007b). Taken together, these findings suggest that supplementation with $\mathrm{NaHCO}_{3}{ }^{-}$has the potential to improve middle-distance running performance. Furthermore, it is possible that the overall "strength" of the effect of $\mathrm{NaHCO}_{3}{ }^{-}$on subsequent performance in the previously mentioned meta-analyses may be underestimated by the inclusion of individuals who suffer from GI issues. Supplementation should therefore be tailored to each individual athlete to determine susceptibility to GI upset and/or body weight gain (fluid retention) and efficacy of supplementation to improve performance.

\section{Further Considerations Regarding Supplements and Elite Middle-Distance Runners}

Beyond the lack of female and/or elite subjects the relative lack of running-based performance studies is also striking (Table 2), as most studies seem to implement cycling-based interventions. However, we would hypothesize that potentially the response of 
some ergogenic aids in elite middle-distance runners may be unique from cyclists. Accordingly, given the very high neuromuscular demands (ground contact times approaching $100 \mathrm{~ms}$ ) coupled with a high anaerobic component (greatest acidosis $\left[\mathrm{H}^{+}\right]$of any event), anything that might improve contractile forces/twitch dynamics, or efficiency of mechanisms associated with muscle contraction, should theoretically improve middle-distance race performance. Therefore, alternative and emerging mechanisms of carnosine (Swietach et al., 2014) and nitrate (Coggan et al., 2018; Whitfield et al., 2017) involving calcium handling, recycling, and/or shuttling, and increasing contractile force and twitch kinetics, requires more research in elite running models, which would notionally accentuate these performance mechanism(s) more than cycling models. Obviously, more performance and mechanistically related ergogenic aid data are required in elite runners. The reader interested in responders versus nonresponders, combinations of ergogenic aids and ergogenic aid periodization should see Peeling et al. (2018) and Stellingwerff et al. (2018).

\section{Conclusion and Future Research Considerations}

Given that middle-distance races are at the crossroads of metabolism, featuring both high aerobic and anaerobic ATP production, there are numerous opportunities for various nutrition interventions to make a significant training and/or race performance impact. Indeed, as an event group, middle-distance athletes might feature the greatest polarization and diversity of training periodization between athletes and between various training seasons, and future nutrition studies should attempt to address this diversity. Indeed, the fiber type and bioenergetics diversity in elite middle-distance athletes requires further nutritional investigation and consideration. Finally, more research on the impact that nutrition has in running/ structural modes of exercise, as compared with the dominate mode of cycling, needs to be addressed. Currently, given the extreme intensities of training and racing in middle-distance athletes, optimizing muscle glycogen contents to support high glycolytic flux (resulting in very high lactate values) with appropriate buffering capabilities, while optimizing power to weight ratios, all in a macro- and microperiodized manner, are the principle nutritional interventions to emphasize.

\section{References}

Astrand, P.O., Hultman, E., Juhlin-Dannfelt, A., \& Reynolds, G. (1986). Disposal of lactate during and after strenuous exercise in humans. Journal of Applied Physiology, 61(1), 338-343. PubMed ID: 3733622 doi:10.1152/jappl.1986.61.1.338

Bailey, S.J., Varnham, R.L., DiMenna, F.J., Breese, B.C., Wylie, L.J., \& Jones, A.M. (2015). Inorganic nitrate supplementation improves muscle oxygenation, $\mathrm{O}_{2}$ uptake kinetics, and exercise tolerance at high but not low pedal rates. Journal of Applied Physiology, 118(11), 1396-1405. doi:10.1152/japplphysiol.01141.2014

Bird, S.R., Wiles, J., \& Robbins, J. (1995). The effect of sodium bicarbonate ingestion on 1500-m racing time. Journal of Sports Sciences, 13(5), 399-403. PubMed ID: 8558626 doi:10.1080/ 02640419508732255

Blancquaert, L., Everaert, I., \& Derave, W. (2015). Beta-alanine supplementation, muscle carnosine and exercise performance. Current Opinion in Clinical Nutrition and Metabolic Care, 18(1), 63-70. PubMed ID: 25474013 doi:10.1097/MCO.0000000000000127
Boorsma, R.K., Whitfield, J., \& Spriet, L.L. (2014). Beetroot juice supplementation does not improve performance of elite $1500-\mathrm{m}$ runners. Medicine \& Science in Sports \& Exercise, 46(12), 23262334. PubMed ID: 24781895 doi:10.1249/MSS.0000000000000364

Buchheit, M., \& Laursen, P.B. (2013). High-intensity interval training, solutions to the programming puzzle: Part I: Cardiopulmonary emphasis. Sports Medicine, 43(5), 313-338. PubMed ID: 23539308 doi:10. 1007/s40279-013-0029-x

Burke, L.M. (2008). Caffeine and sports performance. Applied Physiology, Nutrition, and Metabolism, 33(6), 1319-1334. PubMed ID: 19088794 doi:10.1139/H08-130

Carr, A.J., Hopkins, W.G., \& Gore, C.J. (2011a). Effects of acute alkalosis and acidosis on performance: A meta-analysis. Sports Medicine, 41(10), 801-814. doi:10.2165/11591440-000000000-00000

Carr, A.J., Slater, G.J., Gore, C.J., Dawson, B., \& Burke, L.M. (2011b). Effect of sodium bicarbonate on $\left[\mathrm{HCO}_{3}^{-}\right], \mathrm{pH}$, and gastrointestinal symptoms. International Journal of Sport Nutrition and Exercise Metabolism, 21(3), 189-194. doi:10.1123/ijsnem.21.3.189

Christensen, P.M., Shirai, Y., Ritz, C., \& Nordsborg, N.B. (2017). Caffeine and bicarbonate for speed. A meta-analysis of legal supplements potential for improving intense endurance exercise performance. Frontiers in Physiology, 8, 240. PubMed ID: 28536531 doi:10.3389/fphys.2017.00240

Clarke, N.D., Richardson, D.L., Thie, J., \& Taylor, R. (2018). Coffee ingestion enhances 1-mile running race performance. International Journal of Sports Physiology and Performance, 13(6), 789-794. doi:10.1123/ijspp.2017-0456

Coggan, A.R., Broadstreet, S.R., Mikhalkova, D., Bole, I., Leibowitz, J.L., Kadkhodayan, A., . . . Peterson, L.R. (2018). Dietary nitrate-induced increases in human muscle power: High versus low responders. Physiological Reports, 6(2), e13575. doi:https://doi.org/10.14814/phy2.13575

Costill, D.L., Daniels, J., Evans, W., Fink, W., Krahenbuhl, G., \& Saltin, B. (1976). Skeletal muscle enzymes and fiber composition in male and female track athletes. Journal of Applied Physiology, 40(2), 149-154. PubMed ID: 129449 doi:10.1152/jappl.1976.40.2.149

Currell, K., Conway, S., \& Jeukendrup, A.E. (2009). Carbohydrate ingestion improves performance of a new reliable test of soccer performance. International Journal of Sport Nutrition and Exercise Metabolism, 19(1), 34-46. PubMed ID: 19403952 doi:10.1123/ijsnem.19.1.34

Ducker, K.J., Dawson, B., \& Wallman, K.E. (2013). Effect of beta-alanine supplementation on 800-m running performance. International Journal of Sport Nutrition and Exercise Metabolism, 23(6), 554-561. PubMed ID: 23630039 doi:10.1123/ijsnem.23.6.554

Duffield, R., Dawson, B., \& Goodman, C. (2005). Energy system contribution to 400-metre and 800-metre track running. Journal of Sports Sciences, 23(3), 299-307. PubMed ID: 15966348 doi:10.1080/ 02640410410001730043

Fleck, S.J. (1983). Body composition of elite American athletes. The American Journal of Sports Medicine, 11(6), 398-403. PubMed ID: 6650717 doi:10.1177/036354658301100604

Ganio, M.S., Klau, J.F., Casa, D.J., Armstrong, L.E., \& Maresh, C.M. (2009). Effect of caffeine on sport-specific endurance performance: A systematic review. Journal of Strength and Conditioning Research, 23(1), 315-324. doi:10.1519/JSC.0b013e31818b979a

Gaston, P. (1998). Energetics of high intensity running. Modern Athletics Coach, 36(3), 3-9.

Gladden, L.B. (2004). Lactate metabolism: A new paradigm for the third millennium. The Journal of Physiology, 558(Pt. 1), 5-30. PubMed ID: 15131240 doi:10.1113/jphysiol.2003.058701

Halson, S., Burke, L.M., \& Pearce, J. (2018). IAAF nutrition consensusNutrition for travel-From jetlag to catering. International Journal of Sport Nutrition and Exercise Metabolism. 
Hanley, B., \& Hettinga, F.J. (2018). Champions are racers, not pacers: An analysis of qualification patterns of Olympic and IAAF World Championship middle distance runners. Journal of Sports Sciences, 36(22), 2614-2620. PubMed ID: 29722599 doi:10.1080/02640414. 2018.1472200

Harris, R.C., \& Stellingwerff, T. (2013). Effect of $\beta$-alanine supplementation on high-intensity exercise performance. Nestlé Nutrition Institute Workshop Series, 76, 61-71. doi:10.1159/000350258

Harris, R.C., Tallon, M.J., Dunnett, M., Boobis, L., Coakley, J., Kim, H.J., . . Wise, J.A. (2006). The absorption of orally supplied beta-alanine and its effect on muscle carnosine synthesis in human vastus lateralis. Amino Acids, 30(3), 279-289. PubMed ID: 16554972 doi:10.1007/ s00726-006-0299-9

Hazell, T.J., Islam, H., Townsend, L.K., Schmale, M.S., \& Copeland, J.L. (2016). Effects of exercise intensity on plasma concentrations of appetite-regulating hormones: Potential mechanisms. Appetite, 98, 80-88. PubMed ID: 26721721 doi:10.1016/j.appet.2015.12.016

Heikura, I.A., Burke, L.M., Mero, A.A., Uusitalo, A.L., \& Stellingwerff, T. (2017a). Dietary microperiodization in elite female and male runners and race walkers during a block of high intensity precompetition training. International Journal of Sport Nutrition and Exercise Metabolism, 27(4), 297-304. doi:10.1123/ijsnem.2016-0317

Heikura, I.A., Stellingwerff, T., Mero, A.A., Uusitalo, A.L., \& Burke, L.M. (2017b). A mismatch between athlete practice and current sports nutrition guidelines among elite female and male middle- and longdistance athletes. International Journal of Sport Nutrition and Exercise Metabolism, 27(4), 351-360. doi:10.1123/ijsnem.2016-0316

Heikura, I.A., Uusitalo, A.L.T., Stellingwerff, T., Bergland, D., Mero, A.A., \& Burke, L.M. (2018). Low energy availability is difficult to assess but outcomes have large impact on bone injury rates in elite distance athletes. International Journal of Sport Nutrition and Exercise Metabolism, 28(4), 403-411. doi:10.1123/ijsnem.2017-0313

Hermansen, L., \& Osnes, J.B. (1972). Blood and muscle pH after maximal exercise in man. Journal of Applied Physiology, 32(3), 304-308. PubMed ID: 5010039 doi:10.1152/jappl.1972.32.3.304

Hobson, R.M., Saunders, B., Ball, G., Harris, R.C., \& Sale, C. (2012). Effects of $\beta$-alanine supplementation on exercise performance: A meta-analysis. Amino Acids, 43(1), 25-37. PubMed ID: 22270875 doi:10.1007/s00726-011-1200-z

Impey, S.G., Hearris, M.A., Hammond, K.M., Bartlett, J.D., Louis, J., Close, G.L., \& Morton, J.P. (2018). Fuel for the work required: A theoretical framework for carbohydrate periodization and the glycogen threshold hypothesis. Sports Medicine, 48(5), 1031-1048. PubMed ID: 29453741 doi:10.1007/s40279-018-0867-7

Jentjens, R., \& Jeukendrup, A. (2003). Determinants of post-exercise glycogen synthesis during short-term recovery. Sports Medicine, 33(2), 117-144. PubMed ID: 12617691 doi:10.2165/00007256200333020-00004

Jeukendrup, A.E. (2017). Periodized nutrition for athletes. Sports Medicine, 47(Suppl. 1), 51-63. PubMed ID: 28332115 doi:10.1007/ s40279-017-0694-2

Jones, A.M. (2014). Influence of dietary nitrate on the physiological determinants of exercise performance: A critical review. Applied Physiology, Nutrition, and Metabolism, 39(9), 1019-1028. PubMed ID: 25068792 doi:10.1139/apnm-2014-0036

Jubrias, S.A., Crowther, G.J., Shankland, E.G., Gronka, R.K., \& Conley, K.E. (2003). Acidosis inhibits oxidative phosphorylation in contracting human skeletal muscle in vivo. The Journal of Physiology, 553(Pt 2), 589-599. PubMed ID: 14514869 doi:10.1113/jphysiol. 2003.045872

Martin, D.E., \& Coe, P.N. (1991). Training distance runners. Champaign, IL: Leisure Press.
Maughan, R.J., \& Poole, D.C. (1981). The effects of a glycogen-loading regimen on the capacity to perform anaerobic exercise. European Journal of Applied Physiology and Occupational Physiology, 46(3), 211-219. PubMed ID: 7195804 doi:10.1007/BF00423397

Melin, A., Mountjoy, M., Tenforde, A.S., \& Heikura, I. (2018). Energy availability in athletics: Managing physique needs and high volume training. International Journal of Sport Nutrition and Exercise Metabolism.

Moore, D.R., Robinson, M.J., Fry, J.L., Tang, J.E., Glover, E.I., Wilkinson, S.B., . . . Phillips, S.M. (2009). Ingested protein dose response of muscle and albumin protein synthesis after resistance exercise in young men. The American Journal of Clinical Nutrition, 89(1), 161-168. PubMed ID: 19056590 doi:10.3945/ajcn.2008.26401

O’Rourke, M.P., O’Brien, B.J., Knez, W.L., \& Paton, C.D. (2008). Caffeine has a small effect on 5-km running performance of welltrained and recreational runners. Journal of Science and Medicine in Sport, 11(2), 231-233. doi:10.1016/j.jsams.2006.12.118

Parkhouse, W.S., McKenzie, D.C., Hochachka, P.W., \& Ovalle, W.K. (1985). Buffering capacity of deproteinized human vastus lateralis muscle. Journal of Applied Physiology, 58(1), 14-17. PubMed ID: 3968004 doi:10.1152/jappl.1985.58.1.14

Parolin, M.L., Chesley, A., Matsos, M.P., Spriet, L.L., Jones, N.L., \& Heigenhauser, G.J. (1999). Regulation of skeletal muscle glycogen phosphorylase and PDH during maximal intermittent exercise. The American Journal of Physiology, 277(5, Pt. 1), E890-E900.

Peacock, O., Tjonna, A.E., James, P., Wisloff, U., Welde, B., Bohlke, N., ... Sandbakk, O. (2012). Dietary nitrate does not enhance running performance in elite cross-country skiers. Medicine \& Science in Sports \& Exercise, 44(11), 2213-2219. PubMed ID: 22874535 doi:10.1249/MSS.0b013e3182640f48

Peart, D.J., Siegler, J.C., \& Vince, R.V. (2012). Practical recommendations for coaches and athletes: A meta-analysis of sodium bicarbonate use for athletic performance. Journal of Strength and Conditioning Research, 26(7), 1975-1983. doi:10.1519/JSC.0b013e3182576f3d

Peeling, P., Castell, L., Derave, W., Geyer, H., \& Burke, L.M. (2018). IAAF nutrition consensus-Supplements and sports foodsDRAFT. International Journal of Sport Nutrition and Exercise Metabolism.

Phillips, S.M., Witard, O.C., \& Garthe, I. (2018). IAAF ConsensusProtein for adaptation and physique manipulation in athletics. International Journal of Sport Nutrition and Exercise Metabolism.

Porcelli, S., Ramaglia, M., Bellistri, G., Pavei, G., Pugliese, L., Montorsi, M., . . Marzorati, M. (2015). Aerobic fitness affects the exercise performance responses to nitrate supplementation. Medicine \& Science in Sports \& Exercise, 47(8), 1643-1651. PubMed ID: 25412295 doi:10.1249/MSS.0000000000000577

Price, M.J., \& Simons, C. (2010). The effect of sodium bicarbonate ingestion on high-intensity intermittent running and subsequent performance. Journal of Strength and Conditioning Research, 24(7), 1834-1842. doi:10.1519/JSC.0b013e3181e06e4a

Pugh, L.G. (1971). The influence of wind resistance in running and walking and the mechanical efficiency of work against horizontal or vertical forces. The Journal of Physiology, 213(2), 255-276. PubMed ID: 5574828 doi:10.1113/jphysiol.1971.sp009381

Raysmith, B.P., \& Drew, M.K. (2016). Performance success or failure is influenced by weeks lost to injury and illness in elite Australian track and field athletes: A 5-year prospective study. Journal of Science and Medicine in Sport, 19(10), 778-783. doi:10.1016/j.jsams.2015. 12.515

Reilly, T., Waterhouse, J., Burke, L.M., \& Alonso, J.M. (2007). Nutrition for travel. Journal of Sports Sciences, 25(Suppl. 1), S125-S134. doi:10.1080/02640410701607445 
Sandbakk, S.B., Sandbakk, O., Peacock, O., James, P., Welde, B., Stokes, K., . . . Tjonna, A.E. (2015). Effects of acute supplementation of L-arginine and nitrate on endurance and sprint performance in elite athletes. Nitric Oxide, 48, 10-15. PubMed ID: 25445632 doi:10. 1016/j.niox.2014.10.006

Sandford, G.N., Pearson, S., Allen, S.V., Malcata, R.M., Kilding, A.E., Ross, A., \& Laursen, P.B. (2018). Tactical behaviors in men's 800-m Olympic and World-Championship medalists: A changing of the guard. International Journal of Sports Physiology and Performance, 13(2), 246-249. doi:10.1123/ijspp.2016-0780

Saunders, B., De Salles Painelli, V., De Oliveira, L.F., Da Eira Silva, V., Da Silva, R.P., Riani, L., . . Gualano, B. (2017b). Twenty-four weeks of $\beta$-alanine supplementation on carnosine content, related genes, and exercise. Medicine \& Science in Sports \& Exercise, 49(5), 896-906. doi:10.1249/MSS.0000000000001173

Saunders, B., Elliott-Sale, K., Artioli, G.G., Swinton, P.A., Dolan, E., Roschel, H., . . Gualano, B. (2017a). $\beta$-Alanine supplementation to improve exercise capacity and performance: A systematic review and meta-analysis. British Journal of Sports Medicine, 51(8), 658-669. doi:10.1136/bjsports-2016-096396

Saunders, B., Sale, C., Harris, R.C., \& Sunderland, C. (2014). Sodium bicarbonate and high-intensity-cycling capacity: Variability in responses. International Journal of Sports Physiology and Performance, 9(4), 627-632. PubMed ID: 24155093 doi:10.1123/ijspp. 2013-0295

Shannon, O.M., Barlow, M.J., Duckworth, L., Williams, E., Wort, G., Woods, D., . . O'Hara, J.P. (2017a). Dietary nitrate supplementation enhances short but not longer duration running time-trial performance. European Journal of Applied Physiology, 117(4), 775-785. doi:10.1007/s00421-017-3580-6

Shannon, O.M., McGawley, K., Nyback, L., Duckworth, L., Barlow, M.J., Woods, D., . . O'Hara, J.P. (2017b). "Beet-ing" the mountain: A review of the physiological and performance effects of dietary nitrate supplementation at simulated and terrestrial altitude. Sports Medicine, 47(11), 2155-2169. doi:10.1007/s40279-017-0744-9

Sims, S.T., Rehrer, N.J., Bell, M.L., \& Cotter, J.D. (2007a). Preexercise sodium loading aids fluid balance and endurance for women exercising in the heat. Journal of Applied Physiology, 103(2), 534-541. doi:10.1152/japplphysiol.01203.2006

Sims, S.T., van Vliet, L., Cotter, J.D., \& Rehrer, N.J. (2007b). Sodium loading aids fluid balance and reduces physiological strain of trained men exercising in the heat. Medicine \& Science in Sports \& Exercise, 39(1), 123-130. doi:10.1249/01.mss.0000241639. 97972.4a

Spencer, M.R., \& Gastin, P.B. (2001). Energy system contribution during 200 - to $1500-\mathrm{m}$ running in highly trained athletes. Medicine \& Science in Sports \& Exercise, 33(1), 157-162. PubMed ID: 11194103 doi:10.1097/00005768-200101000-00024

Spriet, L.L. (2014). Exercise and sport performance with low doses of caffeine. Sports Medicine, 44(Suppl. 2), S175-S184. doi:10.1007/ s40279-014-0257-8

Stellingwerff, T. (2018). Case study: Body composition periodization in an olympic-level female middle-distance runner over a 9-year career. International Journal of Sport Nutrition and Exercise Metabolism.

Stellingwerff, T., Anwander, H., Egger, A., Buehler, T., Kreis, R., Decombaz, J., \& Boesch, C. (2012). Effect of two $\beta$-alanine dosing protocols on muscle carnosine synthesis and washout. Amino Acids, 42(6), 24612472. PubMed ID: 21847611 doi:10.1007/s00726-011-1054-4

Stellingwerff, T., Boit, M.K., \& Res, P.T. (2007). Nutritional strategies to optimize training and racing in middle-distance athletes. Journal of Sports Sciences, 25(Suppl. 1), S17-S28. doi:10.1080/ 02640410701607213

Stellingwerff, T., Burke, L.M., \& Morton, J.P. (2018). IAAF-Nutrition consensus-Periodized nutrition for training adaptation. International Journal of Sport Nutrition and Exercise Metabolism.

Stellingwerff, T., \& Cox, G.R. (2014). Systematic review: Carbohydrate supplementation on exercise performance or capacity of varying durations. Applied Physiology, Nutrition, and Metabolism, 39(9), 998-1011. PubMed ID: 24951297 doi:10.1139/apnm-2014-0027

Stellingwerff, T., Maughan, R.J., \& Burke, L.M. (2011). Nutrition for power sports: Middle-distance running, track cycling, rowing, canoeing/kayaking, and swimming. Journal of Sports Sciences, 29(Suppl. 1), S79-S89. doi:10.1080/02640414.2011.589469

Stubbs, R.J., Hughes, D.A., Johnstone, A.M., Horgan, G.W., King, N., \& Blundell, J.E. (2004). A decrease in physical activity affects appetite, energy, and nutrient balance in lean men feeding ad libitum. The American Journal of Clinical Nutrition, 79(1), 62-69. PubMed ID: 14684398 doi:10.1093/ajcn/79.1.62

Swietach, P., Leem, C.H., Spitzer, K.W., \& Vaughan-Jones, R.D. (2014). Pumping $\mathrm{Ca}^{2+}$ up $\mathrm{H}^{+}$gradients: $\mathrm{A} \mathrm{Ca}^{2+}-\mathrm{H}^{+}$exchanger without a membrane. The Journal of Physiology, 592(15), 3179-3188. PubMed ID: 24514908 doi:10.1113/jphysiol.2013.265959

Sygo, J., Kendig, A., Killer, S.C., \& Stellingwerff, T. (2018). IAAFSpecial edition-Nutrition for jumps, throws, and combined events. International Journal of Sport Nutrition and Exercise Metabolism.

Van De Walle, G.P., \& Vukovich, M.D. (2018). The effect of nitrate supplementation on exercise tolerance and performance: A systematic review and meta-analysis. Journal of Strength and Conditioning Research, 32(6), 1796-1808. PubMed ID: 29786633 doi:10.1519/ JSC.0000000000002046

Weyand, P.G., \& Davis, J.A. (2005). Running performance has a structural basis. The Journal of Experimental Biology, 208(Pt. 14), 2625-2631. PubMed ID: 16000532 doi:10.1242/jeb.01609

Whitfield, J., Gamu, D., Heigenhauser, G.J.F., Van Loon, L.J.C., Spriet, L.L., Tupling, A.R., \& Holloway, G.P. (2017). Beetroot juice increases human muscle force without changing $\mathrm{Ca}^{2+}$-handling proteins. Medicine \& Science in Sports \& Exercise, 49(10), 2016-2024. PubMed ID: 28509762 doi:10.1249/MSS.0000000000001321

Wickham, K.A., \& Spriet, L.L. (2018). Administration of caffeine in alternate forms. Sports Medicine, 48(Suppl. 1), 79-91. PubMed ID: 29368182 doi:10.1007/s40279-017-0848-2

Wilkes, D., Gledhill, N., \& Smyth, R. (1983). Effect of acute induced metabolic alkalosis on 800-m racing time. Medicine \& Science in Sports \& Exercise, 15(4), 277-280. PubMed ID: 6312244 doi:10. 1249/00005768-198315040-00004 